\title{
Preparation of Learning Materials for Basic Flute Instrument Based on Locality and ABRSM Curriculum
}

\author{
Robby Ferdian ${ }^{1, *}$, Agung Dwi Putra ${ }^{1}$, Fabio Yuda ${ }^{1}$ \\ 1Department of Drama, Dance and Music Education, Faculty of Language and Art, Universitas Negeri Padang, \\ Padang, Indonesia \\ *Email: robbyferdian@fbs.unp.ac.id
}

\begin{abstract}
The Major Instrument Practice course is a subject that has learning achievements in order to shape student skills in mastering the chosen musical instrument properly and responsibly. Thus, the State University of Padang Music Education Study Program must be able to facilitate it well so that the objectives of the course can be achieved optimally. But the fact is, the Music Education Study Program at Padang State University does not yet have teaching materials that can be used in the learning process of Major Instrument Practices. Therefore, the research team is interested in studying and researching this subject in order to formulate teaching materials into a systematic, easy-to-use, and constructive textbook. The consideration in preparing teaching materials for Major Instrument Practice (Flute) is due to the large number of foreign language teaching materials with varying degrees of difficulty which makes it difficult for students to understand them. The research team believes that the presence of teaching material dictates based on previous considerations is expected to facilitate the learning process of the Flute Practice course. In this study, researchers used an analytical descriptive method that serves to describe or give a picture of the object under study through data or samples that have been collected, this method is believed to be effectively used in this study
\end{abstract} Keywords: major instrument practices course, flute, teaching materials, textbooks, analytical descriptive

\section{INTRODUCTION}

The domain of music education and the music industry moves dynamically in an increasingly competitive and promising direction. The presence of music education study program in 2016 at Universitas Negeri Padang (UNP) is certainly expected to provide quality services in order to produce excellent music scholars.

As one of the new study programs, UNP Music Education Study Program still needs to organize, adapt, and continue to develop its services related to the learning process for students in each course both theoretical and practical courses. Seeing the need for music that is completely instant, the demand for quality music practitioners seems very important. One indicator in assessing the quality of a music academician is if they are able to play musical instruments skillfully.

Based on this, it needs to be examined carefully whether the subject in the Music Education Study Program should immediately receive special attention to be used as a reference in the process of learning an effective practice. In other words, the urgency of the course is worth putting forward without the tendency to rule out other subjects [1]. Of the several subject courses offered at Music Education Study Program at UNP, the subject of expertise related to practical ability is the Major Instrument Practice. This course is divided into several instruments like string, wind (flute, saxophone, trombone, trumpet, clarinet), classical guitar, electric bass, and electric guitar, and drums. The Major Instrument Practice course is a subject whose main objective is to equip students' skills in mastering the musical instrument of their choice. The importance of this course can be seen from the level that is in semester I to semester $\mathrm{V}$ in sequence. if a student fails to pass a certain level then he will not be able to proceed to the next level.

One type of this practice course is the Major Inflatable Instrument Practice (Flute), this course is a course that uses an inflatable flute instrument. Flute is an Inflatable Instrument or referred to as an aerophone. In the previous learning, current course references were only sourced from books in foreign languages (English, German, Italian) so that students had difficulty understanding them. Major Practice Course as an important course in music practice matters certainly must have the ability and facilities to be able to contribute optimally to the ability of student practice, because Music Education study program is a new study program, the availability of teaching materials for this course is not yet available. Therefore, the research team is very interested in studying and researching this teaching material to be able to compile an appropriate textbook.

In this study a teaching textbook will be made containing learning materials consisting of Pieces. Aural Test, Scales and Arpeggio, and Sight Reading based on the ABRSM curriculum and several developments derived from regional song material as an effort to attract students' learning interest 


\section{METHOD}

This study is a qualitative study that aims to produce teaching materials in the form of a textbook that will be used in learning Major Instrument Practice Courses. The approach used in this research is Analytical Descriptive. Descriptive method is a method that functions to describe or give a description of the object under study through data or samples that have been collected as they are without analyzing and making conclusions that are applicable to the public [2]. In other words, the researcher observes the problems found in the previous learning, then the researcher carries out an in-depth analysis of the problem. The results of the analysis are then processed and presented to draw conclusions in formulating the textbook. The data collection technique used is observation.

\section{RESULTS AND DISCUSSION}

\subsection{Flute}

Flute is an oldest musical instrument which is one of the family of aerophone. An aerophone instrument is an instrument whose source of sound comes from air which vibrates metal rods. In modern times the flute is made of a silver/metal base in the form of a long pipe with a diameter $+/-2 \mathrm{~cm}$ divided into 3 pieces. Whereas in the middle era the material used to make flutes was wood, so flutes were categorized as woodwind music instruments.

Flute consists of three parts, namely Head Joint, Body / middle joint, and Foot Joint [3]. These three sections are three different sized pipe rods connected into one section. On the Head Joint there is a Mouth Hole/mouthpiece which is the position of the lips in blowing and ringing it. Then the Body/middle Joint is the position of the fingers in playing the valve/key hole, and the foot joint is the end where several keys are located and this is the shortest pipe. The shape of this instrument can be seen in the following image:

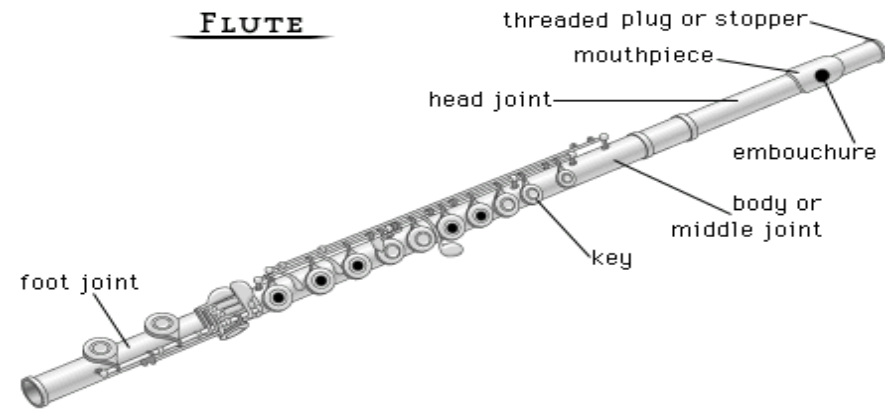

Figure 1 Organology of Flute

Playing musical instruments must be done with fun and the right process, to become a good flute player, students should go through a long process, and not a few beginner flute players who decide to retreat and choose another instrument as an escape. To create and produce a comfortable atmosphere in the training process, a flute player must know the essence of what he is training and what he will achieve with the practice. Often due to Millennials Thought that wants instantaneous, he often forgets the process and only thinks to be able to play one song quickly and a little carelessly, so that often the results of the flute playing are not good and tend not to have a good taste.

\subsection{Basic Competence of Major Flute Instrument Practice}

In terms of formulating the problems that exist in the process of practicing the instrument, researchers think that a flute player must first know the basic competencies that should be the focus when learning the practice of the major flute instrument. The preparation of teaching materials must be in accordance with the basic competencies to be achieved in a learning process. Researchers see flute players often ignore this so that the objectives of the exercise are often not achieved properly. For this reason, researcher tries to identify several important things that should be the aim of the learning process of the flute instrument in order to get the right formula in formulating effective teaching materials. Some basic competencies that must be considered in the practice of Major Flute Instruments are:

\subsubsection{Respiration and Frasering}

When playing the wind instrument, this becomes the most urgent compared to other points, because breathing is the foundation of good frasering. Blowing a flute with the wrong breathing will cause pain in some parts of the body, so that for the good in terms of health and also the game, a flute player must fix breathing techniques first. The breathing that is used in the practice of major flute instruments is diaphragmatic breathing. This breathing is breathing that uses the abdominal cavity as a place to store air [4]. The use of diaphragm cavity is because the diaphragm is the most extensive respiratory cavity to store air and has strong muscles to encourage the blowing.

\subsubsection{Tone Color/Timbre}

Tone Color/timbre is the main identity of the sound. We can recognize instruments through a term known as "timbre". From all over perceptions about music, timbre remains a mysterious thing and cannot be examined with simple mathematics [5]. Timbre is an important aspect of concern in the practice process of flute instruments. This tone color / timbre is very much related to the embacheur in the mouthpiece. 


\subsubsection{Intonation and Articulation}

Intonation speaks of high or low accuracy of sound, while articulation is how to produce clean, clear and true notes. Both of these are of concern because they are the final result of sound production. Articulation in blowing can be done with several syllable pronunciation techniques such as ta, ta-da, tu, tu-ku, etc. This grouping in terms of syllables can be called single tonguing and double tonguing.

\subsubsection{Aural Skills}

Aural is art that is belong in the ear, and sound is the main material [6]. Aural is related to Teaching of pitch and rhythm recognition, which means it is related to the level of sensitivity of a flute player to what is played, be it the tone or rhythm and how the process of interpretation of what is produced in the practice activities of the major instrument flute.

After studying and observing carefully some of the things that are the focus of flute learning above, researchers try to find solutions that can be used as a reference in learning and have clear standard standards in the field of music education. In this case the researchers made the ABRSM Curriculum (Associated Board of the Royal School of Music) as one of the references in making measurable standards in learning flute instruments.

ABRSM is an institution from the UK which is involved in music standardization, ABRSM actively holds examinations in many countries in the world including Indonesia, and certificates from this institution have received recognition from the international music world. The curriculum created by ABRSM is the result of the collaboration of all universities in Europe, this curriculum is revised and developed once every 3 years. By examining the materials contained in the ABRSM curriculum, the researcher sees several interesting things that become strengths in shaping and improving the basic competencies mentioned above.

In addition to using ABRSM as a reference, researchers also explored local wealth by trying to adapt several masterpieces of regional songs that have a unique taste of ornamentations in their horizontal grooves as additional repertoires in the list of piece/song.

Researchers consider taking the element of locality as one of a series of repertoires, because with these materials it is expected that the exercise will become warmer and more attractive through the presence of melodic colors that are familiar in Indonesian culture in a series of musical notations. The learning and introduction of folk songs are usually only taught with an oral system without writing in the form of notation.

\subsection{Locality Curriculum Based on ABRSM Curriculum}

The preparation of Major Instrument Practice course textbooks will contain four elements of material that represent each type of material with the aim of achieving the level of expertise according to the basic competencies above. These four elements are the examination standard of musician certification which is held annually by ABRSM throughout the world, these four elements consist of Scales and arpeggios, Sight Reading, Piece/song and Aural Test.

\subsubsection{Scales and Arpeggios}

Scales are collections of notes in ascending and descending order [7]. Studying the scale is a foundation for some aspects of the technique that will be trained on further material, so that the scale material will be located at the beginning. Knowledge about scale is the initial capital to undergo training and how to interpret training materials properly.

Scale consists of two types, namely major scale and minor scale, both of which have different building intervals. In this dictate the type of scale will be added with some knowledge modes, modes are a series of notes in octaves which form the material of a composition of tones. This mode is a tone scale that is governed by idiomatic expressions [7]. These additions are based on the need for folk songs which contain several modes for their tone choices. Interestingly, folk songs do not only use major minor scales, but if analyzed in depth many contain various modes.

To give a variation on the scales material, this dictate will contain forms other than scale, namely Arpeggios. Arpeggios is almost the same shape as a chord, but because a flute can only play one note at a time, another note is played after the first note at a different level (sequence) so as to produce a chord in a horizontal line.

Scale learning continued with arpeggios is a systematic series, because scale learning must be followed by practicing on arpeggios sequences, meaning that a flute player who has mastered the scale well will easily understand and play arpeggios at various levels.

In this textbook, the scales that will be discussed are simple scales and begin with a scale with a low difficulty level. Scales described here are F Major scale, G major scale, E minor Scale and G Mixolydian and D dorian. The selection of scales is referred to from the ABRSM curriculum plus one mode related to the pieces/song that will be studied in this textbook. After the researcher analyzes the scales, researcher finds that the scale is in the middle register of the flute instrument. So for beginners learning, this is very helpful in long tone practice. With long tone practice, the tone color can be achieved well and more effectively, because it tends to be at the beginning of learning the fluter is still in the process of adjusting the embacheur (lip position) to the mouth hole. 
Here are the forms of the G Major scale, F major scale, E minor Scale, G Mixolydian and D Dorian in the notation:

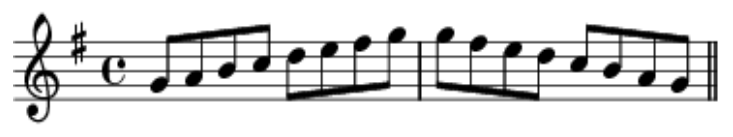

Figure 2 G Major Scale

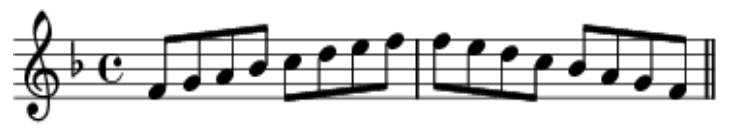

Figure 3 F Major Scale

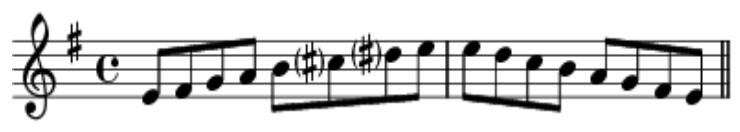

Figure 4 E Minor Scale

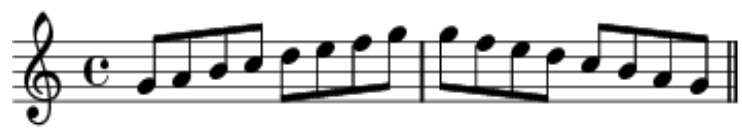

Figure 5 G Mixolydian

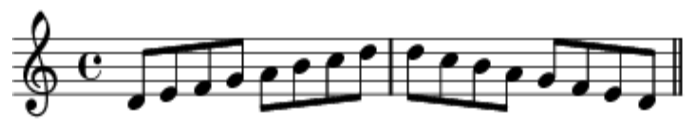

Figure 6 D Dorian

After discussing the scales above, followed by an understanding of the form of arpeggios, the formation of arpeggios is based on the formula obtained from the scales. To expedite the understanding and interpretation of the formation of Arpeggios, it is instructed that in the process of training the scales must be memorized and played without reading the notation, so that automatically all the scales will be attached in memory.

Arpeggios is formed because of the levels in scales. Arpeggios is composed of 3rd (third) intervals starting with the first level (tonica), then continued with the third (median), fifth (dominant) and continued to the eighth (octave) levels. For example, the arpeggio in G Major Scales in one octave is formed by the 1-3-5-8 tones. So the Arpeggio $G$ Major scale pitch arrangement in musical notes is:

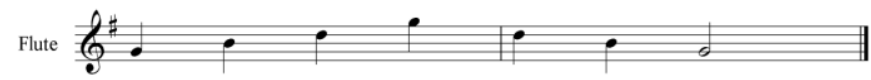

Figure 7 G Major scales Arpeggio

After getting to know the basic form of arpeggios above, a student is taught and required to make variations in the value of the tone and dynamics so that the material becomes varied and gives the ability to be creative in developing the basic Arpeggios material. Forms of development include:
Flute

Figure 8 Arpeggios with Changes in Tone Value

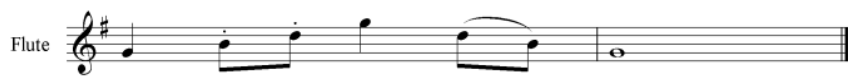

Figure 9 Arpeggios With Changes in Tone Values and Articulation Signs

In conclusion, in this section Scales and arpeggios are the basic ingredients which in practice will be collaborated with diaphragm, long tone, intonation and articulation training methods, so that in this dictate scales and arpeggios will be developed into several forms of tone values, signs of accentuation, and application simple dynamics sign. The diversity of forms is expected to help achieve some fundamental competencies such as good diaphragm practice, strong emburements, good and clear articulation and intonation and increased knowledge of tones on a scale as well as registers.

\subsubsection{Sight Reading}

Sight Reading means the ability to read notations spontaneously/Instantly. Sight Reading requires a fast adaptation process to a new material / sheet, some music scientists often refer to it as Playing by Sight or primavista [8]. Sight reading material contains etudes and melodic compositions/short songs, the formula of this etudes comes from scale and collaboration of arpeggio/broken chord. Sight reading material will be grouped according to the objectives of each etude, some in the form of legato etude, staccato scales, marcato etude, lips slurs, duets, and articulation studies.

One form of etude / short song above is Rakes O Mallow finger twister which is one of the etude originating from the development of scales and broken chords / arpeggios which were developed into a form of short etude. The form of this etude series can be seen in the following notation:

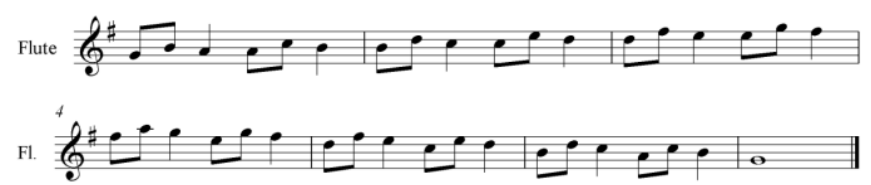

Figure 10 Rakes O Mallow finger Twister

Sight reading material will be focused using classic materials. The purpose of the material in sight reading is the ability to read the notation quickly, precisely and correctly. With practice using etude containing various techniques will make improving reading skills more structured and varied. 


\subsubsection{Pieces/Song}

Pieces/Song is a part of the material developed, in this section unlike western music schools, music courses and various other music education institutions that only contain material from classic songs, reinforcement of material that is local in nature will be more highlighted here. This development is carried out with the consideration that regional songs contain more expressions and accentations that have been infused in daily life, by using these regional songs flutis will be more familiar with the culture and local music accents.

Regional songs that fill the list piece / song will certainly be balanced with song lists that are on classic standard material. So with the variety of lists provided makes a flutist become more enthusiastic in practicing.

The material of the regional song that will be composed is a song that contains local cultural values, especially the Minangkabau culture because the music education program UNP is in the scope of Minangkabau people's lives. The songs in this book are songs that have become regional icons such as Ayam den lapeh, Taktontong, Tanjung Katung, Gundul-Gundul Pacul, Apuse, Minangkabaunese chanting, etc. One form of development of song material from this locality is the Taktontong song which can be seen in the following notation:

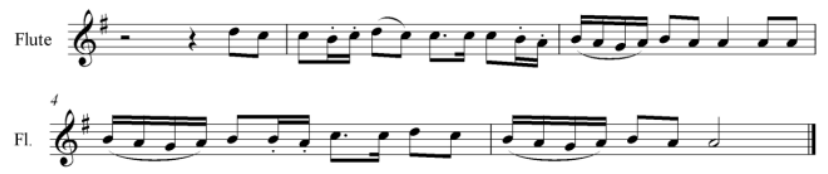

Figure 11 Taktontong Song

The use of locality as a raw material in a new work both in the form of song arrangements and as compositions has already been done by European composers in romantic times such as Bela Bartok [9]. However, the tendency of learning music that exists today in Indonesia still persists with western material that is believed to be the center of world music. Even though Europe apparently has already explored local culture and even eastern culture in search of something new and interesting. By presenting a piece/song with local content in this book, awareness and concern for local music culture is expected to start growing early.

\subsubsection{Aural}

In the ABRSM curriculum aural is one of the points of assessment in each grade-raising test, Aural is closely related to the ability to hear and interpret music, Having a musical ear can have a positive effect on all aspects of music, the better the aural ability, the body will also be more responsive to sounds- sound, Aural is one important point in the formation of a flute player's musicality.

In this Major Flute Instrument Practice dictate containing several aural tests in the practice process and examinations, researchers assume with this aural skill it is possible for musicians to assess the sound or sound of their own instruments and balance with other instruments when playing together, keeping the rhythm and tempo stable, developing musical memory and identify errors yourself. Some Aural practice materials and tests contained in the textbook are:

- Claps a rhythm with a time signature $3 / 4$ and $4 / 4$ read by the lecturer, for example one of these Clapping materials can be seen as the notation below:

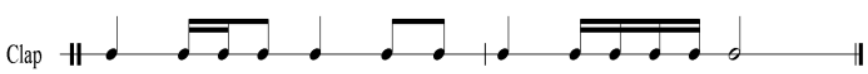

Figure 12 Claps in Aural Practice and Tests

- After the rhythm is played by the lecturer, the student identifies and then repeats it again by explaining the heavy beat and amplifying the pat on the beat. Next, the students mention the type of time signature used;

- Sing a few phrases of the song played by the lecturer. The phrase played starts with a short phrase, then continues until a longer phrase, then mentions a tonic and then counts the phrase that has been played;

- The lecturer plays a song with some changes in the scales (Modulation) path, then the student identifies where the change occurred;

- The last step is the students mentioning the dynamics sign and articulation signs found in the melody path played, then the student repeats with the intonation, articulation and dynamics in the same melody path. The material that will be used in this number is pieces / songs that have local music culture accents, because after researchers have observed that the ornaments found in regional music are very diverse. It is intended that these accentuations can be understood properly. One piece of the melody path for this fourth type can be seen in the following notation:

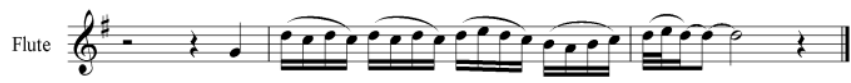

Figure 13 Aural Material with Legato Dynamics and Articulation

\section{CONCLUSION}

The preparation of the Major Instrument Practice textbook is intended to facilitate students in accessing systematic teaching materials. With the presence of this dictate, it is hoped that students will become more organized and focused in the practice process.

After reviewing and observing the existing curriculum at ABRSM, researchers see several advantages that make this curriculum internationally recognized, in terms of newness 
[2] S. P.D, Metode penelitian pendidikan pendekatan kuantitatif.pdf. 2014. then the ABRSM curriculum divides its learning focus into 4 types of competencies that aim to form and developing musicality, namely Scales/Arpeggios, Sight Reading, Pieces/Song, and Aural.

In this textbook researchers adopted 4 focus material contained in the ABRSM and developed in terms of its repertoire. Like in the Scales section, researchers added several modes to increase knowledge of medieval music and as a support in learning pieces/songs of regional songs, in the pieces/songs The researcher song included repertoires of folk songs in addition to the classic ABRSM songs, then in the aural section the researcher added a number of melodic repertoires that contained local music accents.

\section{REFERENCES}

[1] A. D. Putra, I. Epria, D. Putra, and R. Ferdian, "The Development of Music Theory Learning Module in Music Education Program at Faculty of Language and Art Universitas Negeri Padang," J. Pertunjunkan Pendidik. Music, vol. 1, no. 1, pp. 30-40, 2019.
[3] A. Scott, "Flute," pp. 0-14.

[4] S. KarimIda, I. Kaniawati, Y. N. Fauziah, and W. Sopandi, Sistem Pernapasan pada Manusia. Jakarta: Pusat Perbukuan, 2011.

[5] K. Patil, D. Pressnitzer, S. Shamma, and M. Elhilali, "Music in Our Ears: The Biological Bases of Musical Timbre Perception," PLoS Comput. Biol., 2012.

[6] A. Hamilton, "Music and the aural arts," Br. J. Aesthet., 2007.

[7] B. Benward and M. N. Saker, Music in theory and practice. New York: McGraw-Hill, 2008.

[8] A. C. Lehmann and V. McArthur, Sight-Reading. Oxford: Oxford University Press, 2011.

[9] S. Parker, "Béla Bartók's Arab Music Research and Composition," Stud. Musicol., vol. 49, no. 3-4, 2008. 